\title{
Dark State Optical Lattice with a Subwavelength Spatial Structure
}

\author{
Y. Wang (王扬), ${ }^{1, *}$ S. Subhankar, ${ }^{1}$ P. Bienias, ${ }^{1}$ M. Łacki ${ }^{2,3,4}$ T-C. Tsui (崔子俊), ${ }^{1}$ \\ M. A. Baranov, ${ }^{3,4}$ A. V. Gorshkov, ${ }^{1,5}$ P. Zoller, ${ }^{3,4}$ J. V. Porto, ${ }^{1}$ and S. L. Rolston ${ }^{1}$ \\ ${ }^{1}$ Joint Quantum Institute, National Institute of Standards and Technology and the University of Maryland, \\ College Park, Maryland 20742, USA \\ ${ }^{2}$ Jagiellonian University, Institute of Physics, Lojasiewicza 11, 30-348 Kraków, Poland \\ ${ }^{3}$ Institute for Quantum Optics and Quantum Information of the Austrian Academy of Sciences, A-6020 Innsbruck, Austria \\ ${ }^{4}$ Institute for Theoretical Physics, University of Innsbruck, A-6020 Innsbruck, Austria \\ ${ }^{5}$ Joint Center for Quantum Information and Computer Science, \\ National Institute of Standards and Technology and the University of Maryland, College Park, Maryland 20742, USA
}

(Received 1 December 2017; published 20 February 2018)

\begin{abstract}
We report on the experimental realization of a conservative optical lattice for cold atoms with a subwavelength spatial structure. The potential is based on the nonlinear optical response of three-level atoms in laser-dressed dark states, which is not constrained by the diffraction limit of the light generating the potential. The lattice consists of a one-dimensional array of ultranarrow barriers with widths less than $10 \mathrm{~nm}$, well below the wavelength of the lattice light, physically realizing a Kronig-Penney potential. We study the band structure and dissipation of this lattice and find good agreement with theoretical predictions. Even on resonance, the observed lifetimes of atoms trapped in the lattice are as long as $44 \mathrm{~ms}$, nearly $10^{5}$ times the excited state lifetime, and could be further improved with more laser intensity. The potential is readily generalizable to higher dimensions and different geometries, allowing, for example, nearly perfect box traps, narrow tunnel junctions for atomtronics applications, and dynamically generated lattices with subwavelength spacings.
\end{abstract}

DOI: 10.1103/PhysRevLett.120.083601

Coherent control of the position and motion of atoms with light has been a primary enabling technology in the physics of ultracold atoms. The paradigmatic examples of conservative optical potentials are the optical dipole trap and optical lattices, generated by far off-resonant laser fields, with the ac-Stark shift of atomic levels as the underlying mechanism. The spatial resolution for such optical potential landscapes is determined by the diffraction limit, which is of the order of the wavelength of light $\lambda$. This fundamentally limits optical manipulation of atoms. For example, in quantum simulation with atoms in optical lattices, the minimum lattice constant is $\lambda / 2$, setting the energy scale for Hubbard models for both hopping (kinetic energy) and interaction of atoms, with challenging temperature requirements to observe quantum phases of interest [1]. Developing tools to overcome the diffraction limit, allowing coherent optical manipulation of atoms on the subwavelength scale, is thus an outstanding challenge. Following recent proposals [2-4], we report below first experiments demonstrating coherent optical potentials with subwavelength spatial structure, by realizing a KronigPenney-type optical lattice with barrier widths below $\lambda / 50$.

In the quest to beat the diffraction limit, several ideas have been proposed to create coherent optical potentials with subwavelength structure. These include Fourier synthesis of lattices using multiphoton Raman transitions [5,6], optical or radio-frequency dressing of optical potentials [7,8], and trapping in near-field guided modes with nanophotonic systems $[9,10]$ (although they suffer from decoherence induced by nearby surfaces). An alternative approach uses the spatial dependence of the nonlinear atomic response associated with the dark state of a three-level system [11-16] as a means to realize subwavelength atomic addressing and excitation. The subwavelength resolution arises when optical fields are arranged so that the internal dark state composition varies rapidly ("twists") over a short length scale.

As proposed in [3,4], such a subwavelength twist can also be used to create a conservative potential with narrow spatial extent, due to the energy cost of the kinetic energy term of the Hamiltonian $[2,17,18]$. Unlike ac-Stark shift potentials, this twist-induced potential is a quantum effect, with magnitude proportional to $\hbar$. Using this effect, we create 1D lattices with barrier widths less than $\lambda / 50$. This potential realizes the Kronig-Penney (KP) lattice model [19] - a lattice of nearly $\delta$-function potentials. We study the band structure and dissipation and find that the dark state nature of this potential results in suppressed scattering, in good agreement with theoretical models.

Our approach is illustrated in Fig. 1(a). A three-level system is coupled in a $\Lambda$ configuration by two optical fields: a spatially varying strong control field $\Omega_{c}(x)=$ $\Omega_{c} \sin (k x)$ and a constant weak probe field $\Omega_{p}$. The excited 
state $|e\rangle$ can decay to either ground state $\left|g_{i}\right\rangle$. Within the Born-Oppenheimer (BO) approximation, slowly moving atoms in the dark state $\left|E_{0}(x)\right\rangle$ are decoupled from $|e\rangle$, where $\left|E_{0}(x)\right\rangle=\sin (\alpha)\left|g_{1}\right\rangle-\cos (\alpha)\left|g_{2}\right\rangle \quad$ and $\quad \alpha(x)=$ $\arctan \left[\Omega_{c}(x) / \Omega_{p}\right]$ [3]. The two bright states $E_{ \pm}(x)$ have excited state component $|e\rangle$, leading to light scattering. As shown in Fig. 1(b), the fields are arranged in such a way that the dark state changes composition over a narrow region, depending on the ratio $\epsilon=\Omega_{p} / \Omega_{c}$. The kinetic energy associated with this large gradient in the spin wave function gives rise to a conservative optical potential $V(x)$ $[3,4]$ for atoms in $\left|E_{0}(x)\right\rangle$,

$$
V(x)=\frac{\hbar^{2}}{2 m}\left(\frac{d \alpha}{d x}\right)^{2}=E_{R} \frac{\epsilon^{2} \cos ^{2}(k x)}{\left[\epsilon^{2}+\sin ^{2}(k x)\right]^{2}},
$$

where $k=2 \pi / \lambda, E_{R}=\hbar^{2} k^{2} / 2 m$ is the recoil energy, and $m$ is the mass of the atom. The potential $V(x)$ can be viewed as arising from nonadiabatic corrections to the $\mathrm{BO}$ potential $[3,4]$ or artificial scalar gauge potential $[18,20,21]$. When $\epsilon \ll 1$, this creates a lattice of narrow barriers spaced by $\lambda / 2$, with the barrier height scaling as $1 / \epsilon^{2}$ and the full width at half maximum scaling as $0.2 \lambda \epsilon$ [Fig. 1(b)].

The potential $V(x)$ exhibits several properties that distinguish it from optical potentials based on ac-Stark shifts: (1) The explicit dependence on $\hbar$, via the recoil energy $E_{R}$, reveals the quantum nature of $V(x)$ arising from the gradient in the wave function, whereas a typical optical
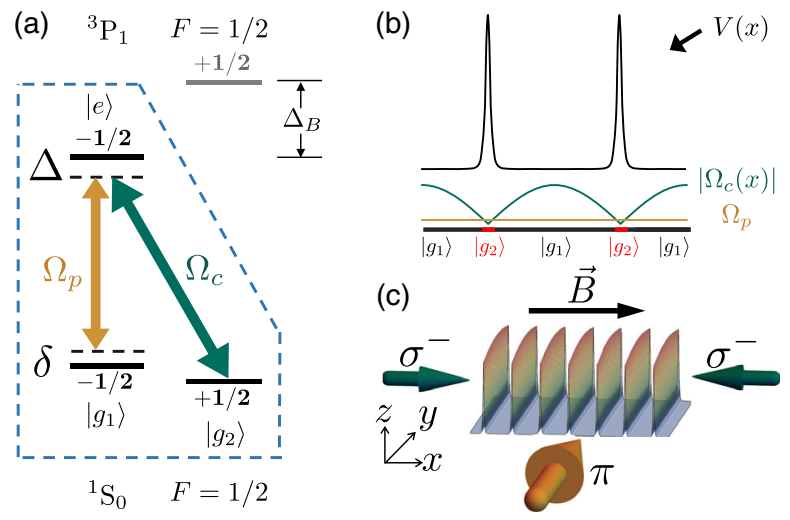

FIG. 1. Level structures and experimental geometry. (a) The three levels in ${ }^{171} \mathrm{Yb}$ used to realize the dark state are isolated from the fourth ${ }^{3} P_{1}, m_{F}=+1 / 2$ state by a large magnetic field. They are coupled by a strong $\sigma^{-}$polarized control field $\Omega_{c}$ (green) and a weak $\pi$ polarized probe field $\Omega_{p}$ (orange). The resulting dark state is a superposition of the ground states $\left|g_{1}\right\rangle$ and $\left|g_{2}\right\rangle$, with relative amplitudes determined by $\Omega_{c}(x) / \Omega_{p}$. (b) Spatial dependence of the dark state composition is created using a standing wave control field $\Omega_{c}(x)$ and a traveling wave probe field $\Omega_{p}$. The geometric potential $V(x)$ (black) arises as the dark state rapidly changes its composition near the nodes of the standing wave. (c) The two counterpropagating $\sigma^{-}$beams creating the standing wave are aligned with a strong magnetic field along $x$, while the $\pi$ beam travels along $y$. potential can be described entirely classically as an induced dipole interacting with the electric field of the laser. (2) Since gradients in wave functions always cost energy, $V(x)$ is always repulsive. (3) The geometric nature of the potential results in it being only dependent on $\epsilon$. By deriving both fields from the same laser, it is relatively insensitive to technical noise. (4) Unlike near-field guided modes $[9,10]$, our scheme works in the far field, thus avoiding the decoherence associated with the proximity of surfaces.

We realize the $\Lambda$ configuration using three states selected from the ${ }^{1} S_{0}, F=1 / 2$ and ${ }^{3} P_{1}, F=1 / 2$ hyperfine manifolds in ${ }^{171} \mathrm{Yb}$. The two ${ }^{1} S_{0}$ ground states $m_{F}= \pm 1 / 2$ comprise the lower two states $\left|g_{1}\right\rangle$ and $\left|g_{2}\right\rangle$ [see Fig. 1(a)]. The ${ }^{3} P_{1}, \quad m_{F}=-1 / 2$ state, with inverse lifetime $\Gamma=2 \pi \times 182 \mathrm{kHz}$, makes up the third state $|e\rangle$ in the $\Lambda$ configuration. The $\left|g_{i}\right\rangle \rightarrow|e\rangle$ transitions are isolated from the transition to the other ${ }^{3} P_{1}, m_{F}=+1 / 2$ state by applying a $12 \mathrm{mT}$ magnetic field $\overrightarrow{\boldsymbol{B}}$ to Zeeman split the two ${ }^{3} P_{1}$ states by $\Delta_{B}=1.8 \times 10^{3} \Gamma$. The same field slightly splits the ${ }^{1} S_{0}$ ground states by $-0.5 \Gamma$ due to the small nuclear magnetic moment. The standing-wave control field $\Omega_{c}(x)$, traveling along $\overrightarrow{\boldsymbol{B}}$, is produced by two counterpropagating $\sigma^{-}$laser beams that couple the $\left|g_{2}\right\rangle$ and $|e\rangle$ states with amplitudes $\Omega_{c 1} e^{i k x}$ and $\Omega_{c 2} e^{-i k x}$. A third beam, $\pi$ polarized and traveling normal to $\overrightarrow{\boldsymbol{B}}$, couples the $\left|g_{1}\right\rangle$ and $|e\rangle$ states with amplitude $\Omega_{p} e^{i k y}$. The frequency of the control and probe beams can be chosen to set the singleand two-photon detunings, $\Delta$ and $\delta$. We define $\delta=0$ as the dark state condition for the isolated three-level system, accounting for the Zeeman splitting. Off-resonant couplings to other states can introduce light shifts, which require nonzero $\delta$ to maintain the dark state condition.

We create an ultracold ${ }^{171} \mathrm{Yb}$ gas in a bichromatic crossed dipole trap by sympathetic cooling with $\mathrm{Rb}$ atoms that are also magnetically confined [22,23]. After $\mathrm{Yb}$ atoms are collected with a temperature of $\simeq 300 \mathrm{nK}\left(T / T_{F}=1.10\right.$, where $T_{F}$ is the Fermi temperature), the magnetic field in the $x$ direction is ramped up in $100 \mathrm{~ms}$ to $12 \mathrm{mT}$, removing $\mathrm{Rb}$ from the trap. The $\mathrm{Yb}$ atoms are then optically pumped into $\left|g_{1}\right\rangle$ using a $50 \mathrm{~ms}$ pulse from one of the control beams, resulting in $\simeq 1.5 \times 10^{5} \mathrm{Yb}$ atoms polarized. The small ${ }^{171} \mathrm{Yb}$ scattering length $\left(-3 a_{0}\right.$ [24], with $a_{0}$ the Bohr radius), plus the lack of $s$-wave scattering in polarized fermions allow us to neglect interactions. The Rabi frequencies of each of the three beams are calibrated by measuring the two-photon Rabi frequencies from $\left|g_{1}\right\rangle \rightarrow$ $\left|g_{2}\right\rangle$ at large $\Delta$ with different pairs of beams. The laser polarization purity and alignment to $\overrightarrow{\boldsymbol{B}}$ are carefully optimized, such that the residual fraction of wrong polarization measured in Rabi frequency is less than $0.5 \%$. To load $\mathrm{Yb}$ into the ground band of the dark state lattice, we first populate the spatially homogeneous dark state by ramping on $\Omega_{c 1}$ followed by $\Omega_{p}$ and then adiabatically 
ramp on $\Omega_{c 2}$ in $1 \mathrm{~ms}$, creating the lattice. We measure the momentum distribution using a band mapping sequence [25], by first ramping off $\Omega_{c 2}$ in $0.5 \mathrm{~ms}$ and then suddenly turning off all the other light fields. We take absorption images after time-of-flight (TOF) along $y$ to measure the momentum along $x$ and $z$; see [26] for further details.

The existence of lattice structure of $V(x)$ leads to Brillouin zones (BZ), visible in TOF images taken after band mapping. Since $k_{B} T$ is less than the band gap, the population is predominantly in the first $\mathrm{BZ}$ and distinct band edges are visible [upper panel in Fig. 2(a)]. The lower panel shows the result with no probe beam, where we find a nearly Gaussian distribution in the lattice direction. We also see nearly Gaussian distributions for atoms loaded in the other two-beam configurations: $\Omega_{c 1}, \Omega_{p}$ and $\Omega_{c 2}, \Omega_{p}$.

For small $\epsilon$, this lattice maps to a 1D KP model. One characteristic feature of the KP lattice is that the energy of the $n$ th-band scales as $n^{2} E_{R}$, such that the band spacing increases with $n$. In contrast, in a deep sinusoidal lattice, the band spacing decreases with $n$. To map out the band structure, we excite atoms from the ground $(s)$ band into the higher bands by shaking the lattice using phase modulation of one of the $\sigma^{-}$beams. After band mapping, we measure the band populations, which become separated after TOF [see Fig. 2(c)]. Figure 2(b) plots the frequency-dependent (a)

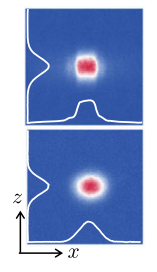

(d)

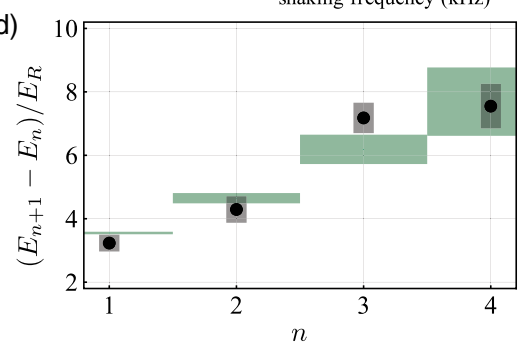

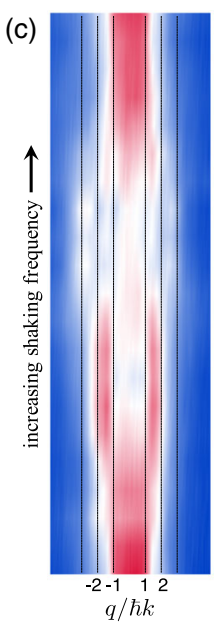

FIG. 2. (a) Band mapping results for atoms loaded into the dark state lattice with three beams (upper) and with only $\Omega_{c}$ beams (lower). The white traces show the integrated momentum distribution in each direction ( $x$ is the lattice direction). (b),(c) Band spectroscopy: in (c), we plot the TOF column density integrated over $z$ after shaking the lattice vs the shaking frequency; in (b), we plot the fraction of the population (frac. pop.) excited to the $p$ band (dark green) and $d$ band (magenta) Brillouin zones [see (c)] vs shaking frequency. Gaussian fits [colored lines in (b)] are used to determine the center frequency and the width of the transition. (d) Band spacing scaling: $E_{n+1}-E_{n}$ is plotted vs the band index $n$ of a dark state lattice with $\Omega_{c}=70 \Gamma, \Omega_{p}=10 \Gamma, \Delta=22 \Gamma$, and $\delta=0$. The gray vertical bars indicate the transition width inferred from the measurements, while the green rectangles are predictions of the expected band spacings and widths [26]. excitation into the first $(p)$ and second $(d)$ excited bands for $\epsilon=0.14$, extracted from the data in Fig. 2(c). The $s \rightarrow d$ excitation arises from a two-step process involving the $p$ band. We map out the band structure up to the $g$ band and plot the energy differences for adjacent bands [see Fig. 2(d)], which increase monotonically with $n$. The green rectangles show the theoretical band spacings and widths, calculated from a model that includes both the light shifts from states outside the three-level system [26] and mixing with the bright states.

Another property of a KP lattice is that, in the deep lattice limit, its band structure is almost independent of the barrier strength (the area under the potential for a single barrier), which scales with $1 / \epsilon$. The band spacings for different $\epsilon$ are plotted in Fig. 3(a) for fixed $\Omega_{c}=100 \Gamma$ and $\Omega_{p}$ varied from 5 to $20 \Gamma$. As expected, the band spacings are almost independent of $\epsilon$, even though the probe power varies by an order of magnitude. The upper panels of Fig. 3(a) show the potentials of the upper bright state (blue) and dark state (green) for three $\epsilon$. For $\epsilon \leq 0.1$, mixing between $E_{0}(x)$ and $E_{ \pm}(x)$ states modifies the band structure, reducing the band spacing. For $\epsilon \simeq 0.1$, we realize a barrier width of $10 \mathrm{~nm}$ with minimal coupling to the bright state. The shaded regions are predictions based on a model that takes bright state couplings into account, which are in better agreement with the measured spacings, compared to the model that has no couplings (dashed line). We attribute the discrepancy between theory and experiment to the residual polarization imperfections, calibration errors in the optical intensity, and limitations of band spectroscopy. We note that the theory predicts a vanishing band width near $\epsilon \simeq 0.125$ and the growth of the bandwidth at even smaller $\epsilon$, due to the interference of dark state and bright state mediated tunneling [26].

Away from $\delta=0$, the state is no longer completely dark and it experiences an additional periodic potential with amplitude $\delta$ [26,30] [Fig. 3(b)]. This additional potential perturbs the KP lattice and the band structure. We verify this by measuring the band spacings as a function of $\delta$ [Fig. 3(b)] and find it agrees with the prediction (shaded area), with the systematic deviation likely coming from the same factors as in Fig. 3(a).

Finally, we study dissipation. The nonadiabatic corrections to the $\mathrm{BO}$ potential that give rise to $V(x)$ also weakly couple the dark state with the bright states, which leads to light scattering, heating the atoms out of the trap. We measure the lifetime $\tau$ in a dark state lattice [Fig. 4(a)] for different $\Delta$ and find it significantly longer for $\Delta>0$ than for $\Delta<0$. This is in contrast to an optical lattice based on ac-Stark shifts, where heating is independent of the sign of $\Delta[31,32]$. To intuitively understand this asymmetry, we use the model described in [4] and note that the coupling to the bright states takes place inside the barrier. An atom can scatter light by admixing with the bright states $E_{ \pm}(x)$ (approximately $\Delta$ independent) or exiting into the energyallowed $E_{-}(x)$ state via nonadiabatic couplings (strongly $\Delta$ 

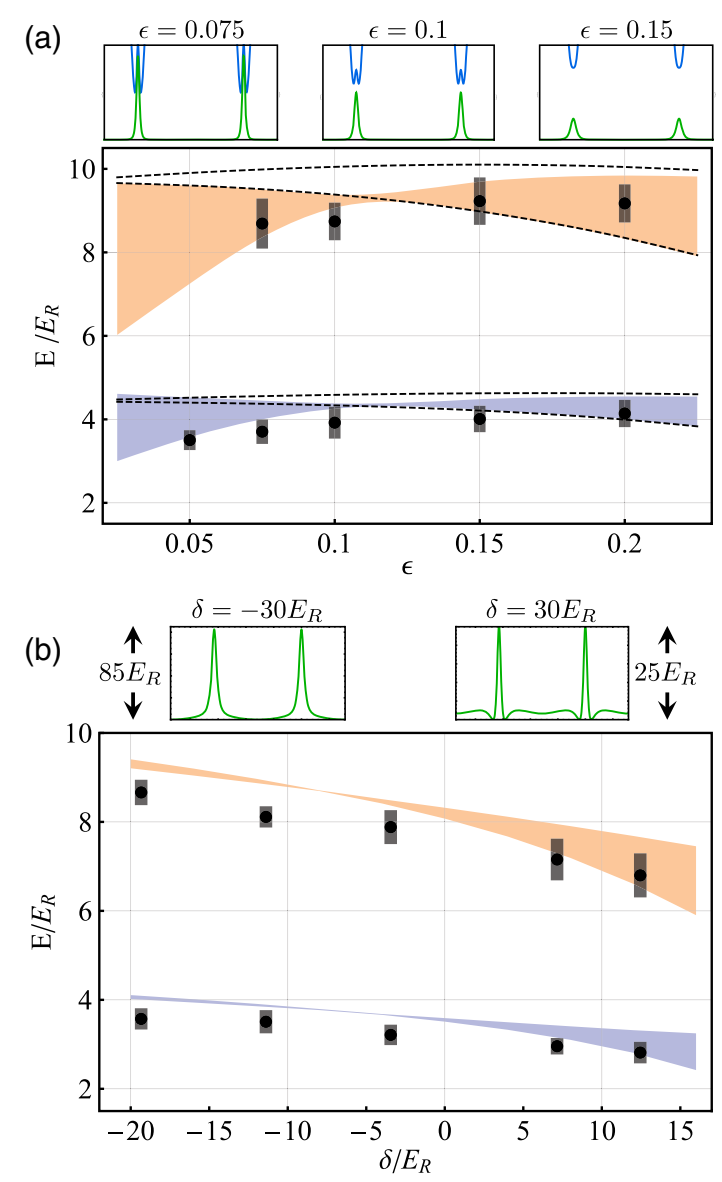

FIG. 3. Band structure scalings. Energies of the $p$ and the $d$ bands with respect to the $s$ band are plotted. (a) Vary $\epsilon$ : $\Omega_{c}=100 \Gamma, \Omega_{p}=5-20 \Gamma, \Delta=22 \Gamma$, and $\delta=0$. Dashed lines indicate the allowed transition energies predicted from modeling $V(x)$ alone, while the shaded regions are from a model including couplings to the bright states. (Upper) Representative potentials for the dark state (green) and bright state (blue). At $\epsilon=0.075$, the bright and dark states are no longer good basis states because of the strong coupling between them. (b) Vary $\delta: \Omega_{c}=70 \Gamma$, $\Omega_{p}=10 \Gamma, \Delta=22 \Gamma$. (Upper) Calculated dark state potentials for positive and negative $\delta$.

dependent). The $E_{-}(x)$ state [red, Fig. 4(a), upper panels] contributes more to the loss, explaining the $\Delta$ asymmetry. The result of the model [26] is depicted as the black line, with an empirical scale factor of 2.2 applied to the theory to account for the unknown relationship between the scattering rate and loss rate $(1 / \tau)$. The lifetime in a homogeneous control field when one of the $\Omega_{c}$ beams is blocked is shown in Fig. 4(a) (inset). The $\tau \simeq 4 \times 10^{5} / \Gamma$ lifetime is almost independent of $\Delta$, as theory would predict, and is $70 \%$ of the expected lifetime due to nonadiabatic coupling to the bright states and off-resonant scattering from states outside the three-level system.

The nonadiabatic bright state coupling also leads to a counterintuitive dependence of the dissipation on the laser power. Figure 4(b) shows the lifetime at constant $\epsilon$ as a (a)

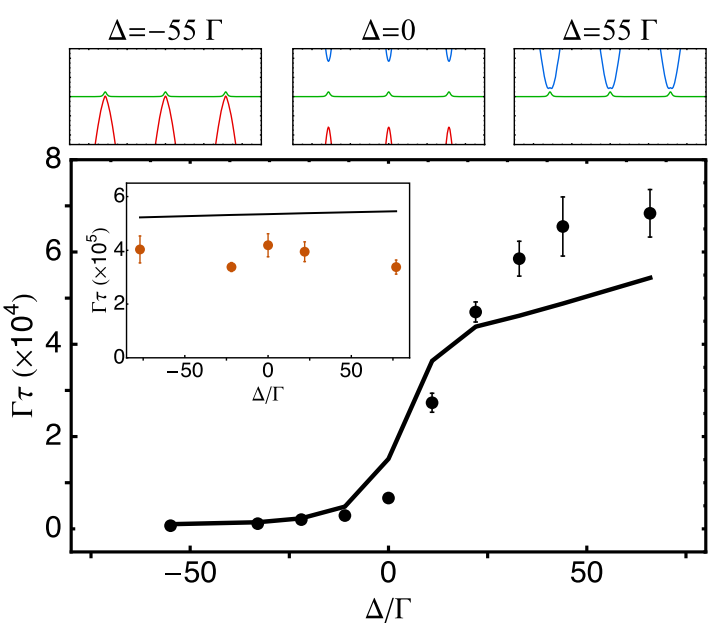

(b)

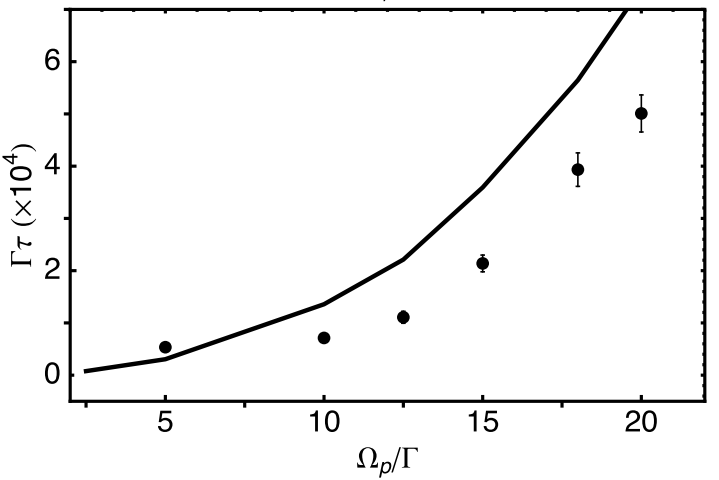

FIG. 4. (a) Lifetime of dark state lattice $\tau$ scaled by the excited state lifetime $\Gamma^{-1}$ vs $\Delta$, with $\Omega_{c}=70 \Gamma, \Omega_{p}=10 \Gamma$, and $\delta=0$. (Inset) Lifetime of the dark state in spatially homogeneous control fields, with $\Omega_{c 1}=35 \Gamma, \Omega_{c 2}=0, \Omega_{p}=10 \Gamma$, and $\delta=0$. (Upper three panels) The two bright state potentials $E_{-}(x)$ (red) and $E_{+}(x)$ (blue), and the dark state potential (green), at different $\Delta$. (b) Lifetime vs $\Omega_{p}$ in a dark state lattice where $\epsilon=0.2$ and $\Delta=0$. The solid black lines are predictions scaled with a factor 2.2 [except for (a) inset, where no scaling is applied]. The error bars represent 1 standard deviation uncertainty from fitting the population decay data.

function of Rabi frequencies. Remarkably, the lifetime increases with Rabi frequency. In contrast, for a regular optical lattice at a fixed detuning, the lifetime does not improve with more laser power. For the dark state lattice, larger $\Omega_{c, p}$ increases the separations between BO potentials, resulting in decreased scattering. In general, the lifetime improves with more laser power and at blue detuning. However, couplings to $E_{+}(x)$ adversely affects the barrier height [similar to the case with $\epsilon \ll 1$ in Fig. 3(a)]. With realistic increase in laser intensity, we can potentially improve the lifetime by an order of magnitude, while maintaining the ultranarrow barriers.

The conservative nanoscale optical potential demonstrated here adds to the toolbox of optical control of atoms, enabling experiments requiring subwavelength motional control of atoms. Such sharp potential barriers could be useful for the creation of narrow tunnel junctions for 
quantum gases [33] or for building sharp-wall box-like traps [34]. In addition, spin and motional localization on small length scales can enhance the energy scale of weak, long range interactions [3]. The dark state lattice is generalizable to 2D and, for example, can be used to study Anderson localization with random strength in the barrier height [35]. By stroboscopically shifting the lattice [36], the narrow barriers should enable optical lattices with spacings much smaller than the $\lambda / 2$ spacing set by the diffraction limit, which would significantly increase the characteristic energy scales relevant for interacting many-body atomic systems.

We thank Victor M. Galitski and Luis A. Orozco for stimulating discussions. Y. W., S. S., T-C. T., J. V. P., and S. L. R. acknowledge support by NSF PFC at JQI and ONR Grant No. N000141712411. P. B. and A. V. G. acknowledge support by NSF PFC at JQI, AFOSR, ARL CDQI, ARO, ARO MURI, and NSF QIS. M. Ł. acknowledges support of the National Science Centre, Poland via Project 2016/23/D/ST2/00721. M. Ł., M. A. B., and P.Z. acknowledge support from the ERC Synergy Grant UQUAM, the Austrian Science Fund through SFB FOQUS (FWF Project No. F4016-N23), and EU FET Proactive Initiative SIQS.

P. B. and M. Ł. contributed equally to this work.

'yuw127@umd.edu

[1] C. Gross and I. Bloch, Science 357, 995 (2017).

[2] R. Dum and M. Olshanii, Phys. Rev. Lett. 76, 1788 (1996).

[3] M. Łącki, M. A. Baranov, H. Pichler, and P. Zoller, Phys. Rev. Lett. 117, 233001 (2016).

[4] F. Jendrzejewski, S. Eckel, T. G. Tiecke, G. Juzeliūnas, G. K. Campbell, L. Jiang, and A. V. Gorshkov, Phys. Rev. A 94, 063422 (2016).

[5] G. Ritt, C. Geckeler, T. Salger, G. Cennini, and M. Weitz, Phys. Rev. A 74, 063622 (2006).

[6] T. Salger, C. Geckeler, S. Kling, and M. Weitz, Phys. Rev. Lett. 99, 190405 (2007).

[7] W. Yi, A. J. Daley, G. Pupillo, and P. Zoller, New J. Phys. 10, 073015 (2008).

[8] N. Lundblad, P. J. Lee, I. B. Spielman, B. L. Brown, W. D. Phillips, and J. V. Porto, Phys. Rev. Lett. 100, 150401 (2008).

[9] A. González-Tudela, C. L. Hung, D. E. Chang, J. I. Cirac, and H. J. Kimble, Nat. Photonics 9, 320 (2015).

[10] M. Gullans, T. G. Tiecke, D. E. Chang, J. Feist, J. D. Thompson, J. I. Cirac, P. Zoller, and M. D. Lukin, Phys. Rev. Lett. 109, 235309 (2012).

[11] A. V. Gorshkov, L. Jiang, M. Greiner, P. Zoller, and M. D. Lukin, Phys. Rev. Lett. 100, 093005 (2008).
[12] M. Kiffner, J. Evers, and M. S. Zubairy, Phys. Rev. Lett. 100, 073602 (2008).

[13] J. A. Miles, Z. J. Simmons, and D. D. Yavuz, Phys. Rev. X 3, 031014 (2013).

[14] M. Sahrai, H. Tajalli, K. T. Kapale, and M. S. Zubairy, Phys. Rev. A 72, 013820 (2005).

[15] J. Cho, Phys. Rev. Lett. 99, 020502 (2007).

[16] K. T. Kapale and M. S. Zubairy, Phys. Rev. A 73, 023813 (2006).

[17] S. K. Dutta, B. K. Teo, and G. Raithel, Phys. Rev. Lett. 83, 1934 (1999).

[18] M. Cheneau, S. P. Rath, T. Yefsah, K. J. Günter, G. Juzeliūnas, and J. Dalibard, Europhys. Lett. 83, 60001 (2008).

[19] R. de L. Kronig and W. G. Penney, Proc. R. Soc. A 130, 499 (1931).

[20] J. Dalibard, F. Gerbier, G. Juzeliūnas, and P. Öhberg, Rev. Mod. Phys. 83, 1523 (2011).

[21] N. Goldman, G. Juzeliūnas, P. Öhberg, and I. B. Spielman, Rep. Prog. Phys. 77, 126401 (2014).

[22] V. D. Vaidya, J. Tiamsuphat, S. L. Rolston, and J. V. Porto, Phys. Rev. A 92, 043604 (2015).

[23] C. D. Herold, V. D. Vaidya, X. Li, S. L. Rolston, J. V. Porto, and M. S. Safronova, Phys. Rev. Lett. 109, 243003 (2012).

[24] M. Kitagawa, K. Enomoto, K. Kasa, Y. Takahashi, R. Ciurylo, P. Naidon, and P. S. Julienne, Phys. Rev. A 77, 012719 (2008).

[25] A. Kastberg, W. D. Phillips, S. L. Rolston, R. J. C. Spreeuw, and P. S. Jessen, Phys. Rev. Lett. 74, 1542 (1995).

[26] See Supplemental Material at http://link.aps.org/ supplemental/10.1103/PhysRevLett.120.083601 for details of experimental setup and calculations on the band structure and lifetime, which includes Refs. [27-29].

[27] S. Subhankar, Y. Wang, A. Restelli, S. L. Rolston, and J. V. Porto (to be published).

[28] J. H. T. Burke, O. Garcia, K. J. Hughes, B. Livedalen, and C. A. Sackett, Rev. Sci. Instrum. 76, 116105 (2005).

[29] D. Steck, Quantum and Atom Optics, available online at http://steck.us/teaching.

[30] P. Bienias et al. (to be published).

[31] J. P. Gordon and A. Ashkin, Phys. Rev. A 21, 1606 (1980).

[32] F. Gerbier and Y. Castin, Phys. Rev. A 82, 013615 (2010).

[33] S. Eckel, J. G. Lee, F. Jendrzejewski, N. Murray, C. W. Clark, C. J. Lobb, W. D. Phillips, M. Edwards, and G. K. Campbell, Nature (London) 506, 200 (2014).

[34] A. L. Gaunt, T. F. Schmidutz, I. Gotlibovych, R. P. Smith, and Z. Hadzibabic, Phys. Rev. Lett. 110, 200406 (2013).

[35] W. Morong and B. DeMarco, Phys. Rev. A 92, 023625 (2015).

[36] S. Nascimbene, N. Goldman, N. R. Cooper, and J. Dalibard, Phys. Rev. Lett. 115, 140401 (2015). 